\title{
In vitro and in vivo Evaluation of the Efficacy and Safety of Powder Hydroxypropylmethylcellulose as Nasal Mucosal Barrier
}

This article was published in the following Dove Press journal:

Medical Devices: Evidence and Research

\author{
Todor A Popov $\mathbb{D}^{1}$ \\ Jean Emberlin ${ }^{2}$ \\ Peter Josling $\mathbb{1 D}^{3}$ \\ Alexander Seifalian (D) ${ }^{4,5}$ \\ 'University Hospital Sv. Ivan Rilski, \\ Department of Occupational Diseases, \\ Sofia, Bulgaria; ${ }^{2}$ Allergy UK, Sidcup, Kent, \\ UK; ${ }^{3}$ Herbal Research Centre, Battle, \\ East Sussex, UK; ${ }^{4}$ NanoRegMed Ltd, \\ BioScience Innovation Centre, London, \\ UK; ${ }^{5}$ Division of Surgery, University \\ College London, London, UK
}

Introduction: Insufflation of powder hydroxypropylmethylcellulose (pHPMC) in the nose has been proven an effective barrier in subjects with rhinitis in many clinical studies. We conducted additionally in vitro and in vivo experiments to address outstanding efficacy and safety issues. Methods: We used an experimental setup to demonstrate the inhibition of the diffusion of allergen extracts (house dust mite, Japanese cedar, Ragweed, Timothy grass) and pollutants (particulate matter $2.5 \mu \mathrm{m}$, PM2.5). Safety of pHPMC when insufflated in the airways of rats was assessed in 24 animals which were sacrificed; tissue sections from lungs, brain and liver were made 1, 24 and 48 hrs after pHPMC inhalation and compared to those of control animals. Results: pHPMC acted as an effective barrier to diffusion of both the liquid allergen extracts and of PM2.5 into the agar covered slides: the quantities of the other tested allergens ranged between $<0.5 \%$ and $14 \%$ of the quantities diffused in the void slides after 6 hrs. The quantity of PM2.5 penetrating the agar was reduced by $94 \%$. Histological photomicrographs did not reveal any evidence of inflammation at 1,24 and $48 \mathrm{hrs}$ after pHPMC insufflation.

Conclusion: Use of pHPMC should be viewed as a barrier enforcing measure against inhalatory ambient intruders.

Keywords: cellulose, hydroxypropylmethylcellulose, HPMC, micronized powder, inhaled allergens, allergen barrier, particulate matter 2.5, PM2.5, safety in rats

\section{Introduction}

Hay fever symptoms are triggered when the nasal mucosa is exposed to allergenic pollen and spores. Previously, numerous products, such as ointments and sprays, have been developed to act as a barrier, but none have been effective. Hydroxypropylmethylcellulose in the form of powder (pHPMC) is a derivative of cellulose, the most abundant polymer in nature. One specific grade of pHPMC, from among the numerous chemical types of cellulose, had been discovered by chance to have the ability to serve as a "mucosal barrier" in the nose. Subsequently, a specially designed system to deliver it into the nose as microcrystalline powder had been patented. ${ }^{1}$ It is licensed as a Class I Medical Device in the EU and most of the world apart from the USA, where it has been registered with the FDA as a Class II Medical Device. ${ }^{2}$ Insufflated nasally, pHPMC swells and forms a gel barrier against offending inhaled noxious or allergenic agents, reducing the nasal symptoms and enhancing the effects of medications for local treatment. ${ }^{3}$ Some thirty clinical studies with pHPMC, which have been performed in patients with allergic rhinitis of different types of sensitization and severity, have provided evidence for the effectiveness and safety of this powder.
Correspondence: Todor A Popov University Hospital Sv. Ivan Rilski, Department of Occupational Diseases, Sofia 1612, Bulgaria

$\mathrm{Tel}+35924519438$

Email ted.popov@gmail.com 
The clinical real-life benefits of pHPMC include creation of a mucosal barrier against offending allergens ${ }^{3}$ with a positive effect on the objective indicators of the disease activity with additional mucosa "healing" properties on top of its "avoidance" effect. ${ }^{4,5}$ It also provides relief on its own in many patients with asthma as a result of the cross-talk between upper and lower airways. ${ }^{6}$ When applied after other nasally applied drugs, pHPMC enhances their effects. ${ }^{7}$ It can also combine under its seal different drugs allowing flexible treatment pending on symptoms and environmental conditions. ${ }^{8,9}$

However, pHPMC is also an effective and safe means of protection against infections, occupational hazards and pollutants, among which particulate matter plays an important role. The aim of the present article was to provide our own unpublished data on the physic-chemical properties of pHPMC, to present our original work performed in three in vitro studies when clinical trials in humans have not been feasible for one reason or another. Another objective was to answer concerns about pulmonary and systemic risks associated with intranasal delivery of pHPMC, for which purpose we conducted a study in rats.

\section{Methods and Materials}

\section{Physico-Chemical Characterization of PHPMC}

We routinely assayed pHPMC batches by laser diffraction technology to obtain average particle size. Particle count and mass distribution were measured in triplicate using a Grimm 1.109 laser particle counter connected to the software Grimm Dust Monitor 3.20. Test-retest reliability was assessed using correlation analysis. It produced a Pearson coefficient of 0.998 and 0.985 .

\section{Supportive Evidence for Efficacy of pHPMC as a Barrier to Offending Agents} A series of experiments based on inhibition of the diffusion of different substances into agar by pHPMC were performed using a similar setup (Figure 1).

\section{In vitro Study I: $\mathrm{pHPMC}$ as Barrier to House Dust Mite Allergen}

The first in vitro proof-of-concept study that measured the diffusion of Dermatophagoides pteronyssinus 1 allergen (Der-p-1, $24 \mathrm{kDa}$ ) through HPMC gel into agar blocks (1.5\%, prepared with $0.9 \%$ saline solution) on microscope slides was done by Diethart et al. ${ }^{10}$ The barrier was prepared by mixing $50 \mathrm{mg}$ of pHPMC with $1 \mathrm{~mL}$ physiological saline

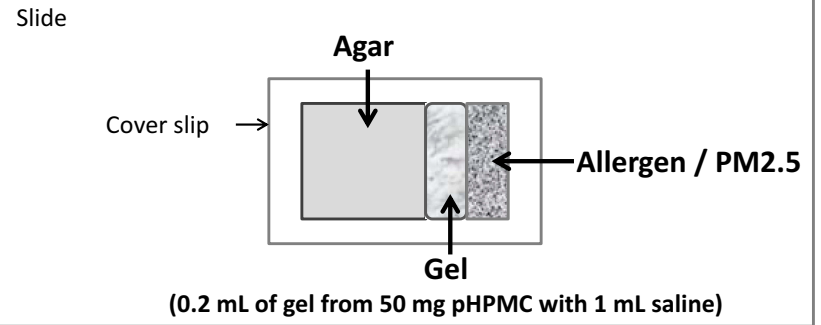

Figure I Graphical presentation of the experimental setup for diffusion inhibition by powder hydroxypropylmethylcellulose (pHPMC), with allergens and particulate matter (PM) $2.5 \mu \mathrm{m}$.

solution to form a $5 \%$ gel, $200 \mu \mathrm{L}$ of which was applied to the open edge of the agar block (Figure 1). After putting a coverslip on top, $20 \mu \mathrm{L}$ of Der p 1 solution $(7.5 \mu \mathrm{g}$ Derp-1 per $1 \mathrm{~mL}$, Alk-Abello) were pipetted on the outer side of the pHPMC gel strip. Slides with agar alone without gel pHPMC were used as controls. All slides were incubated at $35^{\circ} \mathrm{C}$ and $90 \%$ relative humidity to simulate the conditions in the nasal cavity. After 15, 30, 60, 180 and 360 mins slides were taken out of the thermostat, the agar blocks were then carefully removed from the slides and transferred to labeled microtubes containing $0.5 \mathrm{~mL}$ elution medium. The quantities of Der-p-1 were measured by ELISA.

\section{In vitro Study 2: pHPMC as Barrier to Pollen Allergens}

A similar in vitro setup was used to document the dramatic reduction by pHPMC of the diffusion in agar of other common allergens: Cry-j-1, $40 \mathrm{kDa}$ (Japanese cedar), Amb-a-1, 38-40 kDa (Ragweed pollen), ${ }^{11}$ and Phl-p-5, $32 \mathrm{kDa}$ (Timothy grass). Microscope slides with $1.5 \%$ agar were prepared and $100 \mu \mathrm{L}$ HPMC in the form of gel (50 mg pHPMC in $1 \mathrm{~mL}$ physiological saline) were applied as a barrier on one side of the agar, while void slides with agar alone served as controls. Solutions of the allergens were pipetted $(20 \mu \mathrm{L})$ on the opposite side of the HPMC barrier. Slides were made in triplicate for 5 time points (at minutes 15, 30, 60, 180 and 360) for each allergen and were transferred to a chamber mimicking the conditions in the nasal cavity. Samples were eluted and the amounts of allergen in the agar were measured using the respective ELISA kits.

\section{In vitro Study 3: pHPMC as Barrier to Pollutants}

For this, we used the same basic experimental setup with squares of $1.5 \%$ agar mounted on microscope slides and 
pHPMC gel barrier prepared by mixing $50 \mathrm{mg}$ powder with $1 \mathrm{~mL}$ saline applied to one side of the agar. Fluorescent particles in the range of $1.7-2.2 \mu \mathrm{m}, 30 \mu \mathrm{L}$ of 1:50 dilution, were applied to the opposite side of the pHPMC gel barrier. Again, slides were incubated at $35^{\circ} \mathrm{C}$ and $90 \%$ relative humidity for $15,30,60,180$ and 360 mins, after which agar blocks were removed, and the fluorescent particles absorbed into the agar were extracted and counted by flow cytometry. Void slides and slides with lactose gel layer instead of pHPMC served as controls.

\section{Safety Profile}

pHPMC in vivo Study: Insufflation in the Airways of Rats

Despite the theoretical reasoning which suggested a favorable safety profile, the fact that pHPMC is insufflated through the nose led us to perform an animal study. The study received ethics approval of the University College London. All animals were treated with procedures approved by the local governmental animal care committee, and experiments were conducted in accordance with the UK legislation on the protection of animals and the guidelines for the Care and Use of Laboratory Animals. The aim was to document any noxious effects this inert powder might inflict when inhaled through the mouth. ${ }^{12}$ We randomly allocated 24 rats to 4 study groups: Group 1, control/sham group: after anesthetization, the animals were sacrificed and the target organs, lung, liver and brain, were immediately harvested for histology; Group 2: after anesthetization, 3 puffs of pHPMC were delivered, animals euthanized and $1 \mathrm{hr}$ later the target organs were examined histologically; Group 3: as in group 2, the target organs were examined after 24 hrs; Group 4, as in group 2, the target organs were examined 48 hrs later.

Tissue sections from lung, liver and brain were fixed in $10 \%$ neutral buffered formalin, embedded in paraffin, stained with hematoxylin and eosin (H\&E) and examined for the presence of any type of inflammation and necrosis.

During the experiments, systemic hemodynamic data were monitored continuously at a rate of $1 \mathrm{~Hz}$. All hemodynamic data were averaged over 2 min at each observation. All obtained data were also documented and were fed into a commercially available analogue-to-digital data acquisition recording system (MacLab, AD Instruments, Hastings, UK). Values were expressed as mean (SD). Oneway ANOVA with Bonferroni correction was used unless stated otherwise. Student's $t$ test was used for statistical analysis between the groups, $\mathrm{p}<0.050$ was considered statistically significant. Histological H\&E slides were given to an independent person to be read.

\section{Results}

\section{Physico-Chemical Characterization of PHPMC}

The particle size distribution we measured showed that $99.4 \%$ of particles had fallen within the 5 to $500 \mu \mathrm{m}$ diameter range, with a mean particle size of $118 \mu \mathrm{m}$ (Figure 2). The actual particle mass and count distributions proved to be variable with a mean for all particle sizes of $6095.0 \mu \mathrm{g} / \mathrm{m}^{3}$ and a standard deviation of $4709.9 \mu \mathrm{g} / \mathrm{m}^{3}$ for mass distribution (75.4\% of the mean), and a mean of $619,135,967$ counts $/ \mathrm{m}^{3}$ with a standard deviation of $330,964,124$ counts $/ \mathrm{m}^{3}$ for count distribution $(57.5 \%$ of the mean). The particle size distribution of pHPMC is significantly skewed towards larger particles. The pattern of distribution of the pHPMC particles depends on the practical delivery methods utilized to deliver the powder, and on their morphology and swelling behavior due to the hygroscopic nature of cellulose. ${ }^{13}$ Particles of pHPMC are characterized by uneven shape and surface which might affect nasal deposition (Figure 3).

\section{Supportive Evidence for Efficacy of pHPMC as a Barrier to Offending Agents In vitro Study I: PHPMC as Barrier to House Dust Mite Allergen}

In the absence of barrier (control), $72.2 \%$ of the Der-p-1 solution was absorbed after $15 \mathrm{mins}$ and 100\% after 60 mins. In comparison, the presence of pHPMC gel barrier

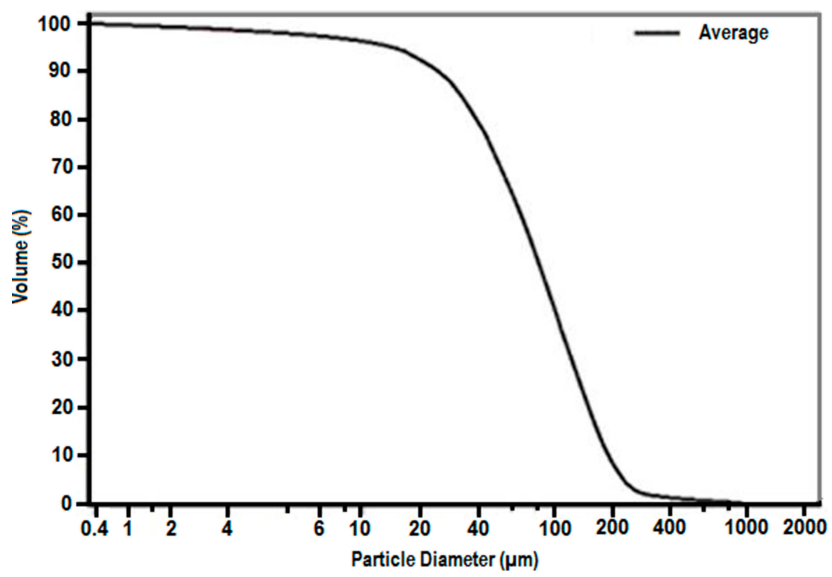

Figure 2 Cumulative volume (average) of successive powder batches over 2 years. It was analyzed by Beckman Coulter LS Particle size analyzer, Philadelphia, USA. 


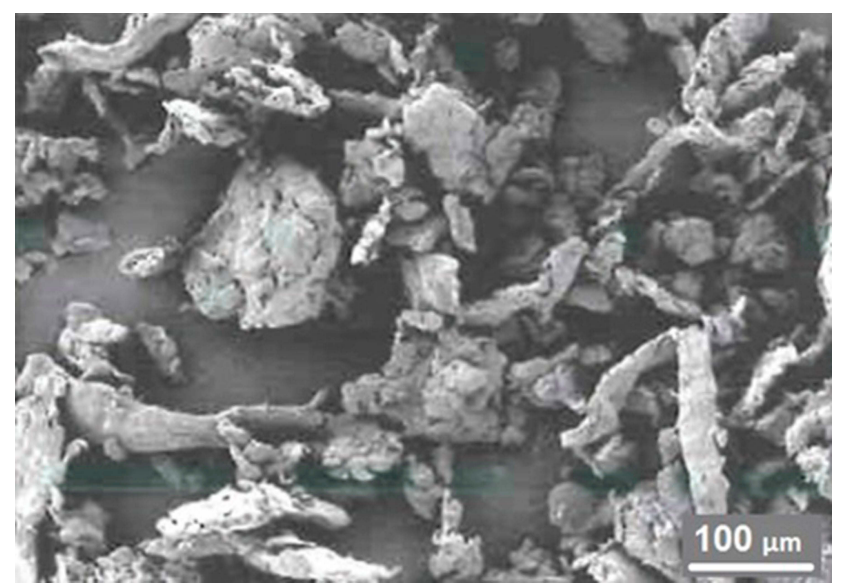

Figure 3 Particles constituting powder hydroxypropylmethylcellulose (pHPMC) in scanning electron microscope (100x magnification) indicates morphology as a key factor of deposition in the airway.

reduced Der-p-1 absorption to $0.76 \%$ after $15 \mathrm{mins}$ and $28.1 \%$ after 360 mins.

In vitro Study 2: pHPMC as Barrier to Pollen Allergens Over the entire time-course of the experiment, the amount of Cry-j-1 absorbed by the agar when pHPMC was in place was $1.39 \%$ of the amount absorbed in the void slides. Similarly, the amount of Amb-a-1 was 3.06\%, and the amount for Phl-p-5 was $<0.5 \%$. The effect was evident as early as 15 mins after the administration of the liquid allergen, supporting the assumption that pHPMC is an effective barrier against Cry-j-1, Amb-a-1 and Phl-p-5 as soon as it is applied and the duration of the effect is at least 360 mins.

\section{In vitro Study 3: pHPMC as Barrier to Pollutants}

With lactose and with no product, the number of fluorescent particles absorbed into the agar increased slowly to reach, at minute 360 , a maximum count of $12,989 \pm 1137$ and $12,999 \pm 465$ [mean $\pm \mathrm{SEM}$ ], respectively. With gel pHPMC as a barrier, the respective numbers were $826 \pm 312$. The reduction by $\mathrm{pHPMC}$ of the $\mathrm{PM}_{2.5}$ uptake into agar was 94\%. This indicates that pHPMC insufflated into the nose has a potent ability to retain and to protect the nasal mucosa from damage by $\mathrm{PM}_{2.5}$, which is a recognized and wellcharacterized pathological factor in all forms of rhinitis. ${ }^{14}$

\section{Safety Profile}

PHPMC in vivo Study: Insufflation in the Airways of Rats

Histological H\&E stained photomicrographs of the lung at magnifications $4,10,20$ and $40 \times$ did not reveal any evidence of inflammation at 1,24 and $48 \mathrm{hrs}$ after challenge and did not differ from the control/sham slides from the rats not subjected to insufflation of pHPMC. There were no significantly different changes between the groups for heart rate, the arterial oxygen saturation, in mean arterial pressure.

\section{Discussion}

Setting a mechanical barrier between the nasal mucosa and the inhalatory hazards of the ambient environment is a simple way to prevent inflammatory reactions in the nose and pHPMC is the only such natural inert compound provided in powder form. Determinants of the deposition of its microcrystals in the nasal cavity are their shape, density, potential electric charges, as well as the individual breathing pattern and airflow rate of the subjects using the product. Particles larger than $5 \mu \mathrm{m}$ are deposited in the nasopharynx, while particle sizes between 1 and $5 \mu \mathrm{m}$, if actively inhaled, can be deposited on the walls of the trachea and bronchial tree. ${ }^{15}$ Particles deposited in the nose and in the tracheo-bronchial airway are trapped in the mucous lining, travel along with it to the pharynx and are swallowed. Only particle sizes below 1 micron could potentially reach the alveoli. In our study, only $0.63 \%$ of the particles were of less than $5 \mu \mathrm{m}$ diameter, and no particles of less than $1.9 \mu \mathrm{m}$ were detected. In other words, essentially none of the pHPMC particles would reach the alveoli; therefore, the whole amount can be considered as swallowed. This kind of spectrum of particle sizes favors the targeted deposition in the nasal cavity in achieving maximal local effect in protecting the mucosa from allergens in allergic rhinitis and any irritants or infectious agents in non-allergic rhinitis.

The ability of pHPMC particles to form a gel when reaching the nasal mucosa is determined by swelling behavior due to the hygroscopic nature of cellulose. ${ }^{13}$ The rough structure of the particles improves swelling by increased contact area which results in more efficient and faster lining of the nasal cavity. ${ }^{16}$ Particle swelling begins immediately upon contact with moisture in the nasal tract and the powder also absorbs moisture from nasal air causing a growth in diameter. This could lead to augmented deposition within the nose which increases in efficiency with increasing particle size. These unique properties offer an explanation as to the role which pHPMC may play in quickly resolving symptoms of seasonal allergic rhinitis.

Our in vitro diffusion experiment with house dust mite allergen clearly demonstrated that the small size of the pHPMC polymer mesh in gel form provides a significant 
mechanical barrier to house dust mite allergens. In patients with perennial allergic rhinitis and sensitization to house dust mite allergen epitopes the reduction of the allergen load on the nasal mucosa down-regulates the chronic inflammation in the nose and due to the interplay and cross-talk between upper and lower airways acts favorably on frequently comorbid asthma. $^{17}$

Our findings in the diffusion experiment with pollens support the clinical findings that pHPMC used by subjects with seasonal allergic rhinitis reduces the hay fever symptoms and the need for pharmacological treatment by preventing the contact between the pollen allergens and the nasal mucosa. ${ }^{3}$

Air pollution is a serious health hazard. Particulate matter about the size of $2.5 \mu \mathrm{m}\left(\mathrm{PM}_{2.5}\right)$ has been shown to cause airway inflammation, to trigger exacerbations of asthma and chronic obstructive pulmonary disease (COPD), to render the lung susceptible to infections, to induce oxidative stress and inflammatory impairments in the cardiovascular and central nervous systems, and to promote the development of diabetes mellitus. ${ }^{18}$ In our diffusion experiment, we demonstrated the capacity of pHPMC to bar the contact of PM2.5 with the nasal mucosa and its influx into the lower airways and the internal milieu of the organism. ${ }^{19}$

The physical and biochemical properties of pHPMC, which is an inert natural product, do not give grounds for safety concerns. Its favorable safety profile has been supported in all clinical studies performed so far, in which no serious and/or severe adverse events have been reported. ${ }^{3}$ A single ex-vivo study suggests that higher doses of cellulose powder may have a negative effect on the viability of the nasal epithelium and on its ciliary beat frequency. ${ }^{20}$ The data of this experimental study hint at a possible negative effect only with the highest concentration of pHPMC. However, these concentrations were just experimental and would never be reached clinically. ${ }^{21}$ However, as it is intended for use by insufflation into the nose, we undertook a thorough characterization of the compound and performed a toxicology study in rats.

Overall, pHPMC is a remarkably safe material when given orally in gram quantities, and the use of Nasaleze in milligram amounts for insufflation in the nose does not present a recognizable risk. Based on the no-observed-adverseeffect level (NOAEL) of $5000 \mathrm{mg} / \mathrm{kg}$ body weight/day from a 90-day feeding study in rats, a tolerable intake for ingestion of pHPMC by humans of $5 \mathrm{mg} / \mathrm{kg}$ body weight/day is accepted, which is more than 100-fold greater than the estimated current consumption of $0.047 \mathrm{mg} / \mathrm{kg}$ body weight/ day. ${ }^{22}$ No studies of genotoxicity or reproductive toxicity have been identified, but the chemistry of the materials, their recognized safety in food use and lack of toxicity in feeding trials does not suggest that further studies are necessary. Actually, one study demonstrated the protective effect of pHPMC against genotoxic agents. ${ }^{23}$ The amount, grade and route of administration of pHPMC used in Nasaleze do not present any serious toxicological risks. In clinical practice, pHPMC is not supposed to be inhaled into the lower airways. Our study and the cited animal study on orally ingested HPMC in rats provide an additional safeguard that even if this happens unintentionally, no harmful consequences are to be expected.

\section{Conclusions}

Our in vitro studies support the capacity of pHPMC to form a gel upon contact with moisture, which provides a reliable barrier to airborne allergens and particulate matter. The in vivo study we did in rats also shows that insufflation of rather high doses of pHPMC through their mouths does not affect the lungs, hearts and livers of the animals.

\section{Expert Commentary}

Precluding the contact between the nasal mucosa and the harmful agents in the ambient environment which attack it (allergens, irritants, microorganisms) is the simplest and most natural approach to prevent triggering inflammatory events in the airways and the ensuing clinical symptoms. This approach is referred to as "barrier-enforcing measures" and may be viewed as a means to achieve allergen avoidance. $^{24}$ Ideally, if implemented properly, this strategy could make the use of any other therapeutic action unnecessary. Attempts have been made to use different substances as barrier enhancers. These include white vaseline, ${ }^{25}$ pollen blocker cream, ${ }^{26}$ lipid-based ointment, ${ }^{27}$ microemulsion, ${ }^{28}$ liposomal formulation ${ }^{29}$ and seawater gel. ${ }^{30}$

Many of the listed approaches could not withstand the test of time and have been abandoned. In contrast, microcrystalline pHPMC has been developed into a patented medical device and licensed in the management of allergic rhinitis. $^{2}$ Its clinical efficacy and real-world effectiveness have been proven in dozens of studies. There had been open questions, which have been taken into consideration and tested in laboratory, in vitro and ex vivo studies. The present overview provides previously unpublished data, which can be of use to the medical and patient communities 
as a basis for wider application of a natural product for the prevention and treatment of airway diseases.

\section{Five-Year View}

Presently, the focus of the application of pHPMC is for prevention and treatment of Allergic Rhinitis. However, it is also effective in preventing infections, and the effects of occupational hazards and pollutants. Targeted in vitro studies may identify new indications for use of pHPMC either on its own or in combination with nasally applied drugs for airway and systemic treatment.

\section{Key Issues}

pHPMC is a cellulose derivative powder with a patented drug delivery system. When insufflated in the nose, it releases a spectrum of particles $99.4 \%$ of which fall within the 5 to $500 \mu \mathrm{m}$ diameter range. pHPMC particles are highly hygroscopic having rough shape and surfaces, which results in fast swelling and gel formation when insufflated in the nose. The HPMC gel layer sets a barrier and prevents the contact of the nasal mucosa with pollen, house dust mite allergens and particulate matter $2.5 \mu \mathrm{m}$ (avoidance effect). In addition to the theoretical arguments and the long-time experience with cellulose derivatives, a study in rats demonstrated that pHPMC does not deposit in the lungs and does not cause adverse systemic effects.

\section{Acknowledgment}

The authors thank Karolina Pomykala for her secretarial help in the preparation of this manuscript.

\section{Disclosure}

The development of hydroxypropylmethylcellulose in the form of micronized powder ( $\mathrm{pHPMC}$ ) is managed and funded by Nasaleze Ltd. Douglas, Isle of Man, IM4 4QE, UK. TA Popov, JC Emberlin, P Josling and A Seifalian have been acting as external scientific advisors to Nasaleze Ltd. and have been involved in conducting clinical studies funded by Nasaleze Ltd. Jean Emberlin reports personal fees from Nasaleze International outside the submitted work. A Seifalian worked for University College London during the study and currently is employed by NanoRegMed Ltd, BioScience Innovation Centre, London, UK. The authors have no other relevant affiliations or financial involvement with any organization or entity with a financial interest in or financial conflict with the subject matter or materials discussed in the manuscript apart from those disclosed.

\section{References}

1. Phadtare D, Phadtare G, Nilesh B, Asawat M. Hypromellose a choice of polymer in extended release tablet formulation. World J Pharmacy Pharmaceut Sci. 2014;3(9):551-566.

2. Product general information Available from: https://www.nasaleze. com/. Accessed 15 Mar 2019.

3. Popov TA, Aberg N, Emberlin J, et al. Methyl-cellulose powder for prevention and management of nasal symptoms. Expert Rev Respir Med. 2017;11(11):885-892. doi:10.1080/17476348.2017.1375408

4. Hristova DM, Kralimarkova TZ, Valerieva A, Popov TA. Objective indicators substantiating the benefits of micronized methylcellulose use by sensitized subjects during the grass pollens season. Allergy. 2017;72(Suppl. 103):729-730.

5. Valerieva A, Popov TA, Staevska M, et al. Effect of micronized cellulose powder on the efficacy of topical oxymetazoline in allergic rhinitis. Allergy Asthma Proc. 2015;36(6):e134-139. doi:10.2500/ aap.2015.36.3879

6. Chen X, Guan WJ, Sun SX, et al. Effects of intranasal cellulose powder on asthma control in children with mild-to-moderate perennial allergic rhinitis: a randomized, placebo-controlled trial. Am J Rhinol Allergy. 2019;33(2):184-193. doi:10.1177/1945892419826509

7. Valerieva A, Church MK, Staevska M, et al. Micronized cellulose powder as a means to enhance intranasal symptoms-driven treatment: patients' attitudes in a real life setting. Allergy. 2016;71(Supplement 102): 13 .

8. Popov TA, Valerieva A, Church M, et al. Real-life study on the effect of micronized cellulose powder as add-on to intranasal as-needed treatment of subjects with pollen allergic rhinitis. J Allergy Clin Immunol. 2016;137(2 Supplement):AB402. doi:10.1016/j.jaci.2015. 12.1246

9. Popov TA, Hristova D, Josling P, Kralimarkova TZ, DuBuske L. Symptom-guided approach for allergic rhinitis treatment augmented with powder methylcellulose sealant. Ann Allergy Asthma Immunol. 2018;121(5- Suppl.):S61-62. doi:10.1016/j.anai.2018.09.203

10. Diethart B, Emberlin JC, Lewis RA. Hydroxypropylmethylcellulose gel application delays Der p 1 diffusion in vitro. Natural Sci. 2010;2 (2):79-84. doi:10.4236/ns.2010.22012

11. Popov TA, Josling P, Church MK. In-vitro setup to test hydroxy-propyl-methyl-cellulose as allergen barrier over a span of 360 minutes. J Allergy Clin Immunol. 2019;143(2 Suppl.):AB193. doi:10.1016/j.jaci.2018.12.592

12. Seifalian A, Birchall M, Bielory L. Investigation of the laryngopharyngeal mucosal distribution of hydroxypropylmethyl cellulose (HPMC) powder after transnasal aerosol application in vivo. Ann Allergy Asthma Immunol. 2012;109(5- Suppl.):A134.

13. Telko MJ, Hickey AJ. Dry powder inhaler formulation. Respir Care. 2005;50(9):1209-1227.

14. Hong Z, Guo Z, Zhang R, et al. Airborne fine particulate matter induces oxidative stress and inflammation in human nasal epithelial cells. Tohoku J Exp Med. 2016;239(2):117-125. doi:10.1620/tjem. 239.117

15. Heyder J. Deposition of inhaled particles in the human respiratory tract and consequences for regional targeting in respiratory drug delivery. Proc Am Thorac Soc. 2004;1(4):315-320. doi:10.1513/pats.200409-046TA

16. Diethart B. The Use of Inert Hydoxypropylmethylcellulose Powder as a Remedy for Allergic Rhinitis. [Chpt 10 " The effect of HPMC application on human nasal cells"]. University of Coventry in collaboration with University of Worcester; 2009.

17. Cruz AA, Popov T, Pawankar R, et al. Common characteristics of upper and lower airways in rhinitis and asthma: ARIA update, in collaboration with GA(2)LEN. Allergy. 2007;62(Suppl 84):1-41. doi:10.1111/j.1398-9995.2007.01551.x

18. Feng S, Gao D, Liao F, Zhou F, Wang X. The health effects of ambient PM2.5 and potential mechanisms. Ecotoxicol Environ Saf. 2016;128:67-74. doi:10.1016/j.ecoenv.2016.01.030 
19. Josling P, Church MK, Popov TA. In vitro study of the adsorption of $2.5 \mathrm{~mm}$ particles(PM2.5) by hydroxy-propyl-methyl-cellulose powder (HPMC). J Allergy Clin Immunol. 2019;143(2 Suppl.):AB24. doi:10.1016/j.jaci.2018.12.074

20. Zhou M, Zuo KJ, Xu ZF, et al. Effect of cellulose powder on human nasal epithelial cell activity and ciliary beat frequency. Int Arch Allergy Immunol. 2019;178(3):229-237. doi:10.1159/000493582

21. Popov TA, Emberlin J, Church MK, Aberg N, Josling P. Powder cellulose in allergic rhinitis management: relevance of in vitro findings to real-life safety. Int Arch Allergy Immunol. 2019;26:1-2.

22. Burdock GA. Safety assessment of hydroxypropyl methylcellulose as a food ingredient. Food Chem Toxicol. 2007;45(12):2341-2351. doi:10.1016/j.fct.2007.07.011

23. Ye J, Zhang $\mathrm{H}$, Wu H, et al. Cytoprotective effect of hyaluronic acid and hydroxypropyl methylcellulose against DNA damage induced by thimerosal in Chang conjunctival cells. Graefe's Arch Clin Exp Ophthalmol. 2012;250(10):1459-1466. doi:10.1007/s00417-012-2087-4

24. Andersson M, Greiff L, Ojeda P, Wollmer P. Barrier-enforcing measures as treatment principle in allergic rhinitis: a systematic review. Curr Med Res Opin. 2014;30(6):1131-1137. doi:10.1185/03007995. 2014.882299

25. Olze H, Wustrow TP, Grigorov A. Provokationstest mit Immerfit Pollenschutzcreme bei Patienten mit allergischer Rhinitis., 2002. Originalia Erfarungsheilkunde (Heidelb). 2002;51:S416-421. doi:10. 1055/s-2002-32436
26. Schwetz S, Olze H, Melchisedech S, Grigorov A, Latza R. Efficacy of pollen blocker cream in the treatment of allergic rhinitis. Arch Otolaryngol Head Neck Surg. 2004;130(8):979-984. doi:10.1001/ archotol.130.8.979

27. Geisthoff UW, Blum A, Rupp-Classen M, Plinkert PK. Lipidbased nose ointment for allergic rhinitis. Otolaryngol Head Neck Surg. 2005;133(5):754-761. doi:10.1016/j.otohns.2005.06. 026

28. Ojeda P, Pique N, Alonso A, et al. A topical microemulsion for the prevention of allergic rhinitis symptoms: results of a randomized, controlled, double-blind, parallel group, multicentre, multinational clinical trial (Nares study). Allergy Asthma Clin Immunol. 2013;9 (1):32. doi:10.1186/1710-1492-9-32

29. Bohm M, Avgitidou G, El Hassan E, Mosges R. Liposomes: a new non-pharmacological therapy concept for seasonal-allergicrhinoconjunctivitis. Eur Arch Oto-Rhino-Laryngol. 2012;269(2):49 5-502. doi:10.1007/s00405-011-1696-6

30. Cingi C, Unlu HH, Songu M, et al. Seawater gel in allergic rhinitis: entrapment effect and mucociliary clearance compared with saline. Ther Adv Respir Dis. 2010;4(1):13-18. doi:10.1177/175346580935 8190
Medical Devices: Evidence and Research

\section{Publish your work in this journal}

Medical Devices: Evidence and Research is an international, peerreviewed, open access journal that focuses on the evidence, technology, research, and expert opinion supporting the use and application of medical devices in the diagnosis, monitoring, treatment and management of clinical conditions and physiological processes. The identification of novel devices and optimal use of existing devices

\section{Dovepress}

which will lead to improved clinical outcomes and more effective patient management and safety is a key feature of the journal. The manuscript management system is completely online and includes a very quick and fair peer-review system. Visit http:// www.dovepress.com/testimonials.php to read real quotes from published authors. 